\title{
Ein Kippbild? Realismus, Idealismus und Husserls transzendentale Phänomenologie ${ }^{1}$
}

Sophie Loidolt

Gewiss sind die Dispute über Realismus und Idealismus in der Phänomenologie manchmal unfruchtbar, manchmal vielleicht sogar ermüdend. Und da das Risiko, Missverständnisse zu erzeugen, eventuell grösser ist, als Klärung zu erlangen, plädieren nicht wenige Autoren dafür, die Begriffe überhaupt zu verabschieden. Dies ist nicht nur heute so. Schon Husserl sah sich in gewissem Sinne jenseits dieser Debatten, ${ }^{2}$ nicht zu reden von Heidegger, ${ }^{3}$ der mit souveräner Geste das gesamte Vokabular der Philosophiegeschichte als missraten zurückwies, darunter auch das der Realismus/Idealismusdebatte. Diese Geste erlaubt, terminologisches, begriffliches, philosophisches Neuland zu betreten. Doch sollte darüber, vor allem von uns "Nachkommen", nicht vergessen werden, dass die Auseinandersetzung mit den keineswegs sinnlosen "grossen Fragen" und traditionellen Antwortlinien, in geschichtlicher und gegenwärtiger Form, auch die Argumentation schärft. Selbst wenn klar ist, dass keine dieser Kategorien und Schubladen so recht "passt«, so ist es doch gewinnbringend zu klären, wo, warum und wie genau sie nicht passen, anstatt sich auf die Einzigartigkeit und Unvergleichbarkeit einer Denkfigur zurückzuziehen.

Mir scheint, dass Husserl explizit diesen Weg gehen wollte, indem er einerseits die radikal neue Herangehensweise seines Ansatzes immer wieder betonte und mit klaren Argumenten ganz eindeutig gewisse Positionen zurückwies (Psychologismus, Repräsentationalismus, metaphysischer Realismus, Objektivismus, Naturalismus) - und andererseits sehr wohl an die Tradition anknüpfte, sowohl terminologisch als auch im Sinne seines ideengeschichtli-

\footnotetext{
1 Dieser Aufsatz ist die überarbeitete und gekürzte Version eines Beitrags (Sophie Loidolt, Ein Kippbild? Realismus, Idealismus und Husserls transzendentale Phänomenologie, 2017, 83-121) in dem fortgesetzten Special Issue `On the Transcendentak in der Zeitschrift Metodo. International Studies in Phenomenology and Philosophy, das mittlerweile drei Heftausgaben umfasst. Ich bedanke mich bei den Herausgebern für die Möglichkeit der Wiederveröffentlichung.

2 Edmund Husserl, Cartesianische Meditationen und Pariser Vorträge, Den Haag 1950, 33f.

3 Martin Heidegger, Sein und Zeit, Tübingen 1967, \$43.
} 
chen Entwicklungsnarrativs in der Krisisschrift. ${ }^{4}$ Er lässt uns auch keineswegs im Unklaren darüber, wie er seinen transzendentalen Idealismus im Verhältnis zu anderen (transzendentalen) Idealismen versteht. ${ }^{5}$ Gleichzeitig stellt er eine intrinsische Verbindung zwischen seiner Phänomenologie und einem transzendentalen Idealismus "grundwesentlich neuen Sinnes" her und sieht letzteren genau in der Durchführung der Phänomenologie verwirklicht. ${ }^{6}$ Ich zitiere eine Passage aus den »Pariser Vorträgen« (1929), die diese Thesen nochmals deutlich illustriert:

"So führt die pure, in reiner Evidenz durchgeführte und dabei in Konkretion durchgeführte Selbstauslegung des ego zu einem transzendentalen Idealismus, aber einem solchen grundwesentlich neuen Sinnes; nicht eines psychologischen Idealismus, nicht eines Idealismus, der aus sinnlosen sinnlichen Daten eine sinnvolle Welt ableiten will, nicht ein Kantischer Idealismus, der mindestens als Grenzbegriff die Möglichkeit einer Welt von Dingen an sich glaubt offenhalten zu können - sondern ein Idealismus, der nichts weiter ist als in Form systematischer egologischer Wissenschaft konsequent durchgeführte Selbstauslegung jedweden Seinssinnes, der für mich, das ego, eben soll Sinn haben können. Dieser Idealismus ist aber nicht ein Gebilde spielerischer Argumentationen, im dialektischen Streit mit Realismen als Siegespreis zu gewinnen. Es ist die an der (dem ego durch Erfahrung vorgegebenen) Transzendenz der Natur, der Kultur, der Welt überhaupt in wirklicher Arbeit durchgeführte Sinnesauslegung, und das ist systematische Enthüllung der konstituierenden Intentionalität selbst. Der Erweis dieses Idealismus ist die Durchführung der Phänomenologie selbst. «"

Nun kann man es auf einen gewissen systematischen "Eklektizismus" des "Allessynthetisierers" Husserls zurückführen, dass sich sowohl »Realisten" als auch "Idealisten" von seiner Phänomenologie angesprochen und bestätigt (oder regelmässig irritiert) finden. ${ }^{8}$ Ich sehe diesen Sachverhalt eher in einer inneren Notwendigkeit

\footnotetext{
4 Edmund Husserl, Die Krisis der europäischen Wissenschaften und die transzendentale Phänomenologie. Eine Einleitung in die phänomenologische Philosophie, Den Haag 1954.

5 Husserl, Cartesianische Meditationen, 192.

6 Vgl. Julia Jansen, On Transcendental and Non-Transcendental Idealism in Husserl - A Response to De Palma and Loidolt, in: Metodo - International Studies in Phenomenology and Philosophy (2017, Special Issue 1.2), 27-39.

7 Husserl, Cartesianische Meditationen, 33f.

8 Jansen, On Transcendental, 25.
} 
seiner Philosophie begründet: Husserl ist gleichzeitig "Realist" und »Idealist", und beides in einem ganz speziellen Sinn. Bei allen Ähnlichkeiten darf man dies aber nicht in derselben Weise wie das Kantische Doppelpaar "empirischer Realismus" - "transzendentaler Idealismus" verstehen: erstens, weil Husserl (wie auch im obigen Zitat noch einmal deutlich zu sehen ist) die Konzeption eines prinzipiell unerfahrbaren "Dinges an sich" zurückweist; und zweitens, weil die Phänomenologie nicht dieselbe strenge Grenzziehung in Bezug auf metaphysische Fragen hat wie Kant. ${ }^{9}$ Manche mögen dies bedauern, Husserl gehört sicherlich nicht dazu:

"Schliesslich möchte ich, um kein Missverständnis aufkommen zu lassen, darauf hinweisen, dass die Phänomenologie, wie wir schon früher ausgeführt haben, nur jede naive und mit widersinnigen Dingen an sich operierende Metaphysik ausschliesst, nicht aber Metaphysik überhaupt, und dass sie nicht etwa die die alte Tradition in der verkehrten Fragestellung und Methode innerlich treibenden Problemmotive vergewaltigt und keineswegs sagt, dass sie vor den 'höchsten und letzten< Fragen halt macht. Das an sich erste Sein, das jeder weltlichen Objektivität vorangehende und sie tragende, ist die transzendentale Intersubjektivität, das in verschiedenen Formen sich vergemeinschaftende All der Monaden. $\|^{10}$

Es gibt keinen Grund, diese klare Aussage auf den letzten Seiten der Cartesianischen Meditationen nicht ernst zu nehmen. Husserls $\mathrm{Zu}-$ rückweisung betrifft nicht metaphysische Thesen an sich, sondern solche, die spekulativ, unanschaulich oder widersinnig sind. Insofern gibt es metaphysische Ansätze, die nicht mit der Phänomenologie vereinbar sind und andere, die nicht nur plausibler sind, sondern sich sogar - wie etwa die transzendentale Intersubjektivität als vergemeinschaftetes Monadenall - in der Durchführung der Phänomenologie selbst ausweisen.

Es würde hier zu weit führen, Husserls Gedanken zur fundamentalen Pluralität und Vergemeinschaftung der Monaden sowie zur Urfaktizität von Ich, Welthabe, intentionalem Ineinander und

\footnotetext{
9 Dies betrifft freilich nur die theoretische Philosophie. Und es muss klar sein, dass man Kant nicht wirklich gerecht wird, wenn man sich bei seiner theoretischen Konzeption bedient, ohne auch mitzubedenken, dass seine praktische Philosophie ganz bewusst über diese Grenzziehung hinausgeht.

${ }^{10}$ Husserl, Cartesianische Meditationen, 182, vgl. $33 f$.
} 
Geschichtsteleologie ${ }^{11}$ nachzugehen. Stattdessen möchte ich auf eine Tendenz in der Debatte antworten, die versucht, dem Realismus Genüge zu tun, indem sie die oben erwähnte Kantische Figur bemüht, nämlich die einer Grenzziehung. Im Vergleich zu Kant möchte ich lediglich darauf hinweisen, dass bei Husserl statt einer Begrenzung eine Entgrenzung unternommen wird. ${ }^{12}$ Husserls transzendentaler Idealismus bleibt damit nicht mehr auf das Erscheinungsfeld der "Dinge für uns" als Feld der einzig erkennbaren Objektivität beschränkt, sondern Erscheinung und Sein werden gleichgesetzt. Damit wird das idealistische Feld entschränkt. Dies bringt einige Herausforderungen mit sich, die uns der Husserl'sche transzendentale Idealismus zu denken aufgibt. Zentral dabei und auch in der wohlwollenden Husserl-Debatte immer wieder kritisch kommentiert, fungieren hier die Absolutheits- und die Abhängigkeitsthese: also die These, dass die Gegebenheitsweise des Bewusstseins eine absolute sei, in der sich reell transzendente, »reale« Gegenstände konstituieren. Husserl hält eindeutig fest (und revidiert dies auch in seinem Spätwerk nicht, wie er in einem Nachwort zu den Ideen I noch 1930 betont ${ }^{13}$ ), dass Bewusstsein "als ein für sich geschlossener Seinszusammenhang zu gelten hat, als ein Zusammenhang absoluten Seins, in den nichts hineindringen und aus dem nichts entschlüpfen kann; der kein räumlichzeitliches Draussen hat und in keinem räumlich-zeitlichen Zusammenhange darinnen sein kann, der von keinem Dinge Kausalität

${ }^{11}$ Ich verweise dafür auf László Tengelyi, Welt und Unendlichkeit. Zum Problem phänomenologischer Metaphysik, Freiburg/München 2014, 185, dem posthum erschienenen Werk, in dem eine eigenständige "Metaphysik zufälliger Faktizität» entwickelt wird. Husserls Metaphysik des vergemeinschafteten Monadenalls habe ich in kürzerer Form in zwei Aufsätzen behandelt: Sophie Loidolt, Zu den metaphysischen Urtatsachen! Das Ineinander der Monaden, in: Phänomenologie und Metaphysik. Phénoménologie \& Métaphysique - Phänomenologische Forschungen, Beiheft 4, hg. v. Alexander Schnell/Inga Römer, Hamburg 2020, 173-190; Sophie Loidolt, Plural Absolutes? Husserl and Merleau-Ponty on Being-In-a-Shared-World and its Metaphysical Implications, in: Empathy, Intersubjectivity, and the Social World - The Continued Relevance of Phenomenology, hg. v. Anna Bortolan/Elisa Magrì, Berlin 2021 (im Erscheinen).

12 Diesen Gedanken habe ich in der "ersten Runde» des Special Issues genauer entwickelt.Vgl. Sophie Loidolt, Transzendentalphilosophie und Idealismus in der Phänomenologie - Überlegungen zur phänomenologischen "Gretchenfrage«, in: Metodo - International Studies in Phenomenology and Philosophy (2015, Special Issue 1.1), 103-135. Der vorliegende Aufsatz ist eine Fortführung dieser Debatte.

${ }_{13}$ Edmund Husserl, Ideen zu einer reinen Phänomenologie und phänomenologischen Philosophie III, Den Haag 1971, 150 f. 
erfahren und auf kein Ding Kausalität üben kann [...].«" Berühmte Phänomenologen wie Maurice Merleau-Ponty $(1966)^{15}$ haben sich schon, trotz aller Affirmation, sehr früh von dieser These distanziert und für eine grundlegende Verwobenheit von Bewusstsein und Welt plädiert. ${ }^{16}$ In einer weiter verfeinerten Lesart, die Husserl bis in die letzten konstitutiven Schichten des inneren Zeitbewusstseins folgt, und etwa von Dan Zahavi ${ }^{17}$ oder Julia Jansen vertreten wird, werden die Absolutheits- und Abhängigkeitsthese ebenfalls relativiert zugunsten einer Reziprozitäts- oder Co-Gegebenheitsthese von »Ich" und "Nicht-Ich«, die eine Art »transzendentale Gestalt« bildet. Julia Jansen, auf deren Argumentation ich mich in diesem Text hauptsächlich beziehen werde, macht den Gedanken stark, dass Husserls genetische Ausführungen zur Hyle als wesentlich ichfremdem Bestand und affizierender Faktizität von Bewusstsein dessen "self-sufficiency" (also Absolutheit) einschränken würden. Damit wäre nicht nur ein Idealismus des "absoluten Bewusstseins" in die Schranken gewiesen, man könnte auch argumentieren, dass "die Welt « oder "das Reale» von Anfang an den Konstitutionsprozess mitbestimmt. ${ }^{18}$ Ich möchte diesen Gedanken im Sinne von Merleau-Ponty zu einer "Gestaltthese " weiterentwickeln und mich dabei fragen, ob dies der zentrale "transzendentale« Gedanke der Phänomenologie sein könnte, der ein Darüber-hinaus-Gehen transzendental-erkenntnistheoretisch verbietet (parallel zu Kants Zusammengehörigkeit von Anschauung und Begriffen). Bei aller Sympathie für diesen Gedanken werde ich schliesslich doch für eine andere Figur zum Verständnis von Husserls transzendentaler Verquickung von Idealismus und Realismus optieren, die ebenfalls von Merleau-Pontys Wahrnehmungsanalysen inspiriert ist, nämlich das Kippbild.

Der Grund dafür ist, dass ich versuche, einen anderen Weg zu finden, als in der Ausdeutung von Husserls transzendentalem Idealismus einen Krypto-Kantianismus Einzug halten zu lassen, um der realistischen Intuition Genüge zu tun. Denn dieser Weg scheint mir mit immanenten Widersprüchen behaftet zu sein. Erstens muss klar sein, dass Husserl selbst einer solchen Deutung widerspricht,

\footnotetext{
${ }^{14}$ Edmund Husserl, Ideen zu einer reinen Phänomenologie und phänomenologischen Philosophie I, Den Haag 1976, 105.

${ }_{15}$ Maurice Merleau-Ponty, Phänomenologie der Wahrnehmung, Berlin 1966.

${ }^{16}$ Vgl. Rudolf Bernet, Transcendental Phenomenology?, in: Phenomenology in a New Key - Between Analysis and History, hg. v. Nicolas de Warren/Jeffrey Bloechl, Dordrecht 2015, 115-133; vgl. Loidolt, Plural Absolutes.

${ }^{17}$ Dan Zahavi, Self-Awareness and Alterity, Bloomington 1999; ders., Husserl's Legacy. Phenomenology, Metaphysics and Transcendental Philosophy, Oxford 2017.

${ }^{18}$ Jansen, On Transcendental, 29.
} 
da sie sich mit einigen Grundeinsichten der Phänomenologie nicht vereinbaren lässt. Zweitens glaube ich, dass man damit eine hartgesottene (metaphysische, spekulative) Realistin ohnehin nicht überzeugen kann. Und drittens denke ich, dass eine mögliche Verwischung des Unterschiedes zwischen "Anerkennung der Faktizität» (Husserl; starker Korrelationismus) ${ }^{19}$ und "Affektionsgrund durch unerkennbare Dinge an sich" (Kant; schwacher Korrelationismus) zu vermeiden ist. Die Realität löst sich bei Husserl (ebenso wie bei Kant) dennoch nicht in Bewusstsein oder Sinnesdaten auf, sie ist bewusstseinstranszendent und sie manifestiert sich in ihrer Objektivität durch eine verleiblichte transzendentale Intersubjektivität (ist also kein "psychologischer Idealismus" wie Husserl dies nennt). ${ }^{20}$ Dies richtig verstehen zu können erfordert einen Wechsel der Einstellungsweise, den ich mit der Figur des "Kippbildes" anzeigen will.

Husserl ist bekannt dafür, von der "natürlichen Einstellung" und dem Wechsel in die "transzendentale Einstellung" zu sprechen, auch die "personale Einstellung" spielt eine grosse Rolle. In $\mathrm{Zu}-$ sammenhang mit seiner Monadenlehre spricht Husserl oft von "absoluter Einstellung" oder "absoluter Welt-Interpretation «. ${ }^{21}$ Was er damit meint, ist im Grunde nichts als eine ganz konsequente Art, alles in der Relation des "für" zu denken, des Dativs des Erscheinens. ${ }^{22}$ Das heisst: nie naturkausal zu denken, auch nicht in der

19 Dieser Ausdruck und die Einteilung in "starken« und "schwachen" Korrelationismus gehen auf Quentin Meillassoux, Nach der Endlichkeit, Berlin 2008, zurück (vgl. Loidolt, Transzendentalphilosophie).

20 "Solange man nur die psychologische Subjektivität kennt und sie absolut setzt und doch die Welt als ihr blosses Korrelat erklären will, ist der Idealismus widersinnig, ist er psychologischer Idealismus - derjenige, den der ebenso widersinnige Realismus bekämpft. [...] Aber da der Kontrast zwischen psychologischer und transzendentaler Subjektivität ungeklärt blieb und der vorherrschende englische Sensualismus oder Naturalismus Konstitution von Realem nicht als eine intentionale, Sinn und wahres Sein für die transzendentale Subjektivität ergebende Leistung verständlich machen konnte, so blieb für die Folgezeit der unfruchtbare und unphilosophische, auf dem natürlichen Boden sich bewegende Streit zwischen Idealismus und Realismus im Gang, und es blieb herrschend die unzulängliche Interpretation des Sinnes, den die grossen Idealisten eigentlich intendierten, freilich, wie gesagt, ohne für sich und für andere den radikalen Unterschied dieses Sinnes als eines transzendentalen im Gegensatz zum psychologischen zur Abhebung bringen zu können." (Husserl, Ideen III, 154) Wie hier deutlich wird, ist ein wesentlicher Knackpunkt für ein korrektes Verständnis von Husserls transzendentalem Idealismus seine antirepräsentationalistische Intentionalitätstheorie, worauf hier nur verwiesen werden kann (vgl. dazu Loidolt, Transzendentalphilosophie).

${ }^{21}$ Edmund Husserl, Zur Phänomenologie der Intersubjektivität. Texte aus dem Nachlass. Zweiter Teil: 1921-1928, Den Haag 1973, 244.366.

22 Vgl. Loidolt, Zu den metaphysischen Urtatsachen; vgl. dies., Plural Absolutes. 
natürlichen Einstellung, sondern jede Kausalität, auch psychophysische, als erlebte Kausalität zu denken. Was bringt ihn dann dazu, neben der lebensweltlichen Perspektive noch diese "metaphysische» anzusetzen, die seiner Auffassung nach dem Lebensweltrealismus keinesfalls widerspricht, sondern ihn nur endgültig transzendental expliziert? Ich denke, es könnte dieses Motiv sein: Wenn Bewusstsein nicht reduzibel ist auf Natur (genauso übrigens wie Natur nicht reduzibel ist auf Bewusstsein, deshalb aber auch nicht absolut ist), wenn es irreduzibles Für-(sich-)Sein ist, dann muss eine Beschreibung rein von Bewusstseinsperspektive her so aussehen, dass sie alles intentional nachvollziehen kann. Husserls »Idealismus" ist also eine Art von Erklärungsform (für ihn die höchste Form derVerstehbarkeit als radikale Sinnesklärung ${ }^{23}$ ) und keine Substanz- oder gar Letztbegründungstheorie im Sinne der metaphysischen Letztbegründung von notwendigem Sein. In der Metapher des Kippbildes kann das Geist-Welt-Verhältnis je so oder so interpretiert werden.

Im Folgenden möchte ich näher auf Jansens ${ }^{24}$ Aufsatz On Transcendental and Non-Transcendental Idealism in Husserl in der Zeitschrift Metodo eingehen, die in einem mehrere Nummern umfassenden Schwerpunkt namens 〉On the Transcendentak eine intensive Debatte zwischen verschiedenen Husserl-Interpret:innen anregte. Jansens Argumentation lautet (ich formuliere ein wenig überspitzt), dass Husserl zuerst das Bewusstsein hypothetisch als absolut angesetzt habe, die späten transzendental-genetischen Untersuchungen aber gezeigt hätten, dass es doch nicht so absolut sei: "[T]he independence of consciousness from reality qua objectivity does not make consciousness strictly self-sufficient; nor is reality epistemologically or ontologically redundant. $\ll^{25}$ Das Argument für diese Auslegung beruht hauptsächlich auf der Angewiesenheit des Bewusstseins auf eine ichfremde Hyle (was sich in den genetischen Untersuchungen zeige), wobei diese Hyle nicht empiristisch verstanden werden darf, sondern als das, worauf sich ein Akt wesenhaft beziehen muss, um überhaupt Akt/Noesis/Bewusstsein von ... sein zu können. Weiters bringt Jansen Zitate von Husserl, die auf die apriorische Zusammengehörigkeit von gegenständlichem Sinn und Bewusstsein ${ }^{26}$

\footnotetext{
${ }^{23}$ Husserl, Die Krisis, 180; Edmund Husserl, Zur Phänomenologie der Intersubjektivität. Texte aus dem Nachlass. Dritter Teil: 1929-1935, Den Haag 1973, 370f.

${ }^{24}$ Jansen, On Transcendental.

25 Ebd., 29.

${ }^{26}$ Edmund Husserl, Transzendentaler Idealismus. Texte aus dem Nachlass (19081921), Dordrecht 2003, 73; Jansen, On Transcendental, 29. Mir scheint allerdings gerade dieses Zitat in seinem Kontext besonders stark auf Husserls Idealismus hinzuweisen: »Was ist, muss sich zur Gegebenheit bringen lassen; jeder mögliche Gegenstand,
} 
verweisen, sowie darauf, dass ein konkretes Ich immer einen Kern von Nicht-Ich im Sinne seiner hyletischen Bestände hat. ${ }^{27}$ Dieses weise auf einen "nichtsubjektiven Kern" in allem Subjektiven hin.

Was besagt nun diese Argumentation genau und auf welche Schlüsse muss sie uns führen? Sehen wir uns dies noch einmal im Detail in folgenden Schritten an: (1) Zuerst möchte ich klären, was Husserl mit seinem transzendentalen Idealismus nicht meint. (2) Dann werde ich seine Aussagen zur Zusammengehörigkeit von Ich und Nicht-Ich genauer analysieren und eine Interpretation dazu vorlegen, die klar den Unterschied zu einer Kantischen "Widerlegung des Idealismus« hervorhebt. (3) Schliesslich stelle ich die Frage nach der transzendentalen Figur der "Gestalt" von Ich/Nicht-Ich. (4) Den Abschluss bildet eine Betrachtung der Faktizität in der transzendentalen Phänomenologie und ihr Verhältnis zur Vereinbarkeit von Idealismus und Realismus.

\section{Was ist Husserls transzendentaler Idealismus nicht?}

Zunächst scheint es mir wichtig zu klären, welche Thesen Husserls transzendentaler Idealismus nicht impliziert. Dies ist deshalb unabdingbar, weil einige Befürchtungen, die bei einer idealistischen These im Raum stehen, auf Husserls Ansatz schlichtweg nicht zutreffen. Husserls Idealismus impliziert in keiner Phase seines Denkens die Behauptungen:

- „Es gibt nur absolutes Bewusstsein. « Husserl dagegen: »als ob man sagen wollte, alles andere Sein sei nur ein scheinbares, ein unwirklicher Schein, ein Fiktum. Das wäre freilich grundfalsch. «"

- "Natur ist bloss phänomenalistisch aufzufassen. "Husserl dagegen: "zu sagen, die Naturwissenschaft habe es nicht mit der Natur zu tun, die wahren Objekte, mit denen sie sich beschäftigt, seien Empfindungen, und was wir Dinge, Atome etc. nennen, seien blosse Symbole, denkökonomische Abkürzungen für Empfindungen und

das Wort im weitesten Sinn (verstanden), hat als Korrelat eine mögliche Anschauung [...]. So gehört also a priori unaufhebbar Gegenstand, gegenständliches Sein und Bewusstsein zusammen."

${ }^{27}$ Husserl, Transzendentaler Idealismus, 73; Jansen, On Transcendental, 29.

${ }^{28}$ Husserl, Transzendentaler Idealismus, 70. 
Empfindungszusammenhänge, das ist der Gipfel derVerkehrtheit. ${ }^{29}$

- „Bewusstsein schafft sich seine Welt, wodurch es keinen Raum für Passivität gibt. Gleichbedeutend mit:Alles ist deduzierbar aus dem transzendentalen Ego." Husserl dagegen: "Das Urphänomen hinsichtlich der Welterfahrung, der Welterkenntnis [...] ist der Heraklitische Fluss der subjektiven Welthabe, des subjektiv vorgegebenen Weltlichen $[\ldots]$. . $^{30}$

Uwe Meixner ${ }^{31}$ streicht richtigerweise Husserls Anti-Reduktionismus in Bezug auf die gegebene natürliche Realität hervor, d.h. dass Natur sich nicht in Bewusstsein auflösen lässt und deshalb jeder Phänomenalismus zurückzuweisen ist. Husserl ging es nie darum, einen Idealismus zu vertreten, »der sozusagen die Materie totschlägt, der die erfahrene Natur für blossen Schein erklärt und nur das seelische Sein für das wahre erklärt«. ${ }^{32}$ Dies hält er nicht nur für "verkehrt«, ${ }^{33}$ sondern für "grundfalsch «, ${ }^{34}$ und dies über sein ganzes Werk hinweg.

Ich denke, es ist absolut zentral, sowohl an dieser These festzuhalten als auch an Husserls dazugehöriger These, dass Bewusstsein »die Wurzel und in einem anderen Bild - die Quelle alles dessen [ist], was sonst noch 'Sein heisst und heissen kann ${ }^{35}$ (beides, so habe ich an anderer Stelle ${ }^{36}$ argumentiert, ergibt sich aus Husserls Verständnis der Intentionalität und widerspricht sich nicht). Diese beiden Thesen und ihre Verbundenheit scheinen mir die Herausforderung für ein Verständnis von Husserls transzendentalem Idealismus zu sein. Noch einmal anders zusammengefasst: Der Gegenstand gibt sich selbst und kein Bild von ihm. Seine z.B. physikalischen Beschaffenheiten sind keinesfalls auf Bewusstsein (psychische oder mentale "Eigenschaften «) oder blosse Sinnesphantome zu reduzieren. Das Ding, die Natur, das Physikalische ist, was es ist, und nichts anderes. Trotzdem wist" es nur, insofern es sich im Bewusstsein manifestiert und ausweist.

\footnotetext{
${ }^{29}$ Ebd., 71.

${ }^{30}$ Edmund Husserl, Späte Texte über Zeitkonstitution (1929-1934). Die C-Manuskripte, Dordrecht 2006, 1.

${ }^{31}$ Uwe Meixner, Husserls transzendentaler Idealismus als Supervenienzthese - ein interner Realismus, in: Husserl und die Philosophie des Geistes, hg. v. Manfred Frank/ Niels Weidtmann, Berlin 1980, 178-208.

${ }^{32}$ Edmund Husserl, Einleitung in die Philosophie. Vorlesungen 1922/23, Dordrecht $2002,276$.

${ }^{33}$ Ebd.

${ }^{34}$ Husserl, Transzendentaler Idealismus, 70.

35 Ebd.

${ }^{36}$ Loidolt, Transzendentalphilosophie.
} 
Was bedeutet dies genauer? Dazu eine längere Passage Husserls aus dem Band Transzendentaler Idealismus:

»Setzung von Natur scheint gar keine Setzung von Bewusstsein notwendig zu fordern. In Wahrheit ist es aber so, dass Setzung von Bewusstsein mit seinem gesamten Gehalt die Setzung aller Natur und alles sonstigen Seins überhaupt implicite schon in sich fasst. Aber auch Setzung von Natur (ist) in gewisser Weise Setzung von Bewusstsein. Aber freilich, da liegt die Sache nicht so, dass aus dem Inhalt der Natur (des Gesetzten) Bewusstsein irgendwie zu entnehmen wäre.Vielmehr werden (wir) erst von der Erkenntnis der Natur zurückgeführt auf Bewusstsein und von da zur Erkenntnis, dass, wenn Bewusstsein nicht wäre, nicht nur Erkenntnis nicht möglich wäre, sondern auch Natur selbst jeden Anhalt, ihre Wurzel, ihre arché verlieren würde und damit ein Nichts wäre.

Dagegen: Setzung von Bewusstsein ist so geartet, dass wir aus dem Inhalt, dem Wesen des Bewusstseins das Sein entnehmen können. Bewusstsein ist seinem Wesen nach in mannigfachen Gestaltungen, die seinen Bestand ausmachen, auf >Gegenständer gerichtet. Hinblick auf das Wesen des Bewusstseins führt notwendig darauf, dass es sein Leben und Sein ausmacht, Gegenstände zu meinen und gemäss den oder jenen aktuellen Motivationen Gegenstände zu bestimmen als wahre wirkliche Naturgegenstände: Gegenstände, die sind, was sie sind, nur in

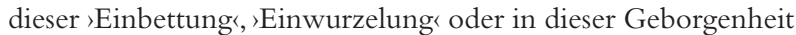
im absoluten Bewusstsein. ${ }^{37}$

Husserl geht es also um ein reflexives Unternehmen, das für Abhängigkeit aber gleichzeitig auch für die Irreduzibilität von Realem/ Natur in Bezug auf Bewusstseinskorrelationen argumentiert. Dies läuft auf eine Seinsfundierung hinaus, die weder als materialer Aufbau (wie bei einem Haus), noch als kausale Implikation, noch als irgendeine Fundierung innerhalb desselben Seinsbereichs (z.B. wie bei fundierenden Akten) verstanden wird. Die "Supervenienzbeziehung « - wenn wir so sprechen wollen, ${ }^{38}$ - in Husserl'scher Prägung ist vielmehr so zu verstehen, dass alles, was "Sein" in der Natur bedeuten kann, korrelative Bewusstseinsgestaltungen impliziert (und zwar motiviert mögliche, die auf wirkliche zurückverweisen und nicht bloss logisch mögliche). Naturgegenstände »sind « nur so, wie Husserl sagt, nämlich »eingebettet«, "eingewurzelt« in korrelative

${ }^{37}$ Husserl, Transzendentaler Idealismus, 71f.

${ }^{38}$ Meixner, Husserls transzendentaler Idealismus. 
Bewusstseinsgestaltungen. Umgekehrt benötigt Bewusstsein diese "Einbettung« nicht bzw. bettet es sich, wenn man so will, selbst ein.

\section{Zu Ich und Nicht-Ich als Figuren der genetisch- transzendentalen Analyse der Monade}

Im Folgenden möchte ich nun auf einige Zitate eingehen, die die notwendige Zusammengehörigkeit von Ich und Nicht-Ich thematisieren. Zunächst ist festzustellen, dass Husserl diese Analysen oft im Kontext der Frage durchführt, was zum vollen monadischen Sein gehört. ${ }^{39}$ Es geht ihm um die "volle Konkretion des Ich", das "pure Ich" ohne Gehalt seiner strömenden Gegenwart ist für Husserl mabstrakt ${ }^{40}{ }^{40}$ Insofern betont Husserl, dass das konkrete Ich in seinem Leben als Bewusstseinsleben beständig einen Kern von Hyle, von Nicht-Ich, hat, der aber "wesentlich ichzugehörig" ist: ${ }^{41}$

"Zu jeder Monade gehört Einheit eines Ich, über die ganze Zeitdauer erstreckte Identität des Ich mit allem Ichlichen, ferner Ichfremdes und doch 'Subjektives`, ein notwendiger ichfremder Bereich der Monade. Also ein durch die immanente Zeit hindurch erstreckter Bereich von hyletischen Gegenständen und evtl. ein in solchen immanenten Gegenständen sich erscheinungsmässig darstellender Bereich von transzendent gesetzten Gegenständen. Die vergangene Hyle schreibt in der Monade der künftigen keine Wesensnotwendigkeiten des Kommens in ihrer Bestimmtheit vor. Die Hyle kommt zufällig, sie steht nur unter den allgemeinsten Wesensgesetzen kontinuierlicher Zeitfüllung. ${ }^{42}$

Zum Kern dessen, was eine Monade ausmacht, ein absolutes Bewusstsein, gehört also Ichfremdes, Passives, etwas das hingenommen wird und keinesfalls produziert oder kreiert wird: »Keine Hyle [...] kann ich erfinden, ich muss sie erfahren haben. ${ }^{43}$ Gleichzeitig ist dieses Ichfremde reell immanent, d.h. es ist keineswegs als ein VonAussen-Affizierendes, Transzendentes misszuverstehen. "Ichfremd« heisst daher keineswegs nicht bewusstseinszugehörig. Das Bewusstsein besteht nicht nur aus "Ich". Es ist nicht ein Ende oder ein Pol des Gegebenheitsgeschehens, dem ein Anderes gegenüberstünde, sondern

${ }^{39}$ Husserl,Zur Phänomenologie der Intersubjektivität (ZweiterTeil), 14.30.45f.51f.244.

${ }^{40}$ Husserl, Späte Texte, 53.

${ }^{41}$ Husserl, Zur Phänomenologie der Intersubjektivität (Zweiter Teil), 379.

${ }^{42}$ Ebd., 14.

43 Ebd., 113. 
es ist dieses Gegebenheitsgeschehen selbst, mit seinen ichlichen und ichfremden Momenten, mit Passivität und Aktivität,Vorgegebenheit und Betätigung daran (Husserl nennt dies manchmal "Ur-Ich«). Dies reicht bis in die tiefsten Zeitanalysen hinein:

"Das Subjektive ist Bewusstsein von Nichtsubjektivem, und die Zeitigung ist auch Zeitigung dieses Nichtsubjektiven. Der nichtsubjektive Kern in Originarität, in der Konkretion untrennbar vom Subjektiven, als worin es wechselnd rerscheint $[\ldots]$. . $^{44}$

Die Frage ist nun: Wie ist dies genau zu verstehen? Ist damit die Nicht-Absolutheit des Bewusstseins dargelegt? Zeigt der "nichtsubjektive Kern", dass es Bewusstsein ohne das es Affizierende gar nicht geben kann? (»Ohne ein Reich der Vorgegebenheiten, ein Reich konstituierter Einheiten, konstituiert als Nicht-Ich, ist kein Ich möglich. ${ }^{45}-$ auf dieses Zitat bezieht sich Jansen. ${ }^{46}$ ) Und: Haben wir damit den Hinweis darauf, dass Husserl doch daran glaubt, dass es eine "fertige Welt" oder irgendeine Art von Welt, Aussen, Ding an sich etc. gibt, die uns qua »Vorgegebenheit« affiziert?

Ich zerlege und kommentiere im Folgenden zwei Zitate, woran ich diese Fragen näher erörtern will:

"Ich bin<. [...] Ich finde mich als Ichpol, als Zentrum von Affektionen und Aktionen, ich finde mich dabei bezogen auf eine reale Umwelt. Eidetisch sehe ich aber ein, dass ich als Pol nicht denkbar bin ohne eine reale Umgebung. Das Ich ist nicht denkbar ohne ein Nicht-Ich, auf das es sich intentional bezieht; ...

Bis hierher scheint es, dass Husserl Ich und Welt, ${ }^{47}$ verstanden als die real-raumzeitlich konstituierte Welt als gleichsam vorrangig ("finde mich bezogen auf") oder zumindest gleichrangig ("Ich nicht denkbar ohne Nicht-Ich") ansetzt. Aber welchen Status hat die Vorgegebenheit, die ich eidetisch einsehe? Ist mit dem wesensmässig notwendigen Nicht-Ich, das wesensmässig meine Icherfahrung als Welthabe ausmacht, schon eine "fertige Welt» gemeint?

... dabei ist noch nicht gesagt, dass dies Nicht-Ich eine reale raumzeitlich-kausale Welt, eine Natur ist, und es ist auch nicht gesagt, dass dieses Ich andere Ich sneben« sich hat oder auch nur haben könnte.

\footnotetext{
${ }^{44}$ Husserl, Späte Texte, 361.

${ }^{45}$ Husserl, Zur Phänomenologie der Intersubjektivität (Zweiter Teil), 379.

${ }^{46}$ Jansen, On Transcendental, 30.

${ }^{47}$ Dies bezieht immer die Intersubjektivität mit ein in derTrias "Ich - Welt - Andere«, die aber hier nicht zur Erwähnung kommt.
} 
Husserl verneint dies offensichtlich. Was bedeutet dann aber die wesentliche Zusammengehörigkeit von Ich und Nicht-Ich, wenn sie nicht einmal eine reale Welt garantiert?

"Aber jedenfalls in der reinen Innenbetrachtung, in der Erwägung des Sinnesgehaltes eines ego cogito, ego sum in Abwandlung seiner erdenklichen Möglichkeiten finde ich, dass Ich und Nicht-Ich untrennbar sind, dass Ich undenkbar ist denn als sich bin bewusst', und jedenfalls bewusst eines $>$ Realen, , mag ich auch ein blosses Gewühl von Empfindungsdaten sein. « $^{48}$

Mir scheint hier folgende Interpretation naheliegend: Bewusstsein, auch absolutes Bewusstsein, ist, im Gegensatz zu so vielen Interpretationen von Husserls Werk, grundlegende Offenheit. Auch Offenheit für eine Affektion blossen Gewühls (und das ist ein weitaus grösseres und radikaleres Zugeständnis an die Macht der Faktizität als Kant dies jemals machen kann). Bewusstsein ist also niemals ein abgekapselter Bereich. Es ist nicht nur dann "weltoffen", wenn sich auch eine kohärent konstituierbare Welt zeigt. Es ist auch »offen«, wenn es dies nicht tut. ${ }^{49}$ Sehen wir uns noch eine eher erkenntnistheoretische Formulierung an, die dieselbe Figur aufgreift:

"Kein Gegenstand ist als Wirklichkeit denkbar ohne die wirkliche Subjektivität, die befähigt ist, diesen Gegenstand in wirklichem Erkennen zu realisieren. Man kann sehr wohl sagen: kein Objekt ohne Subjekt wie kein Subjekt ohne Objekt, wo Objekt Gegenstand in weitestem Sinne besagt. Die Subjektivität, das konkrete Ego, macht davon keine Ausnahme, ...

Dies klingt zweifellos sehr (neo-)Kantianisch. Aber ist es das wirklich? So wie Kant es in der "Widerlegung des Idealismus" versteht, dass ich ein "Aussen" annehmen muss, dass Bedingung für die Kohärenz meines Erlebens ist, sicher nicht. ${ }^{50}$ Denn was Husserl mit »Gegenstand in weitestem Sinne" meint, impliziert nur, dass Wirklich-Sein

\footnotetext{
${ }^{48}$ Husserl, Zur Phänomenologie der Intersubjektivität (Zweiter Teil), 244.

${ }^{49}$ Husserl nennt dies ungewöhnlicherweise sogar - unter Anführungszeichen - das "Reale", weil "ich bin bewusst" schlichtweg heisst: Sein ist präsent, sei es auch nur das von wirren Empfindungsdaten und meinem Empfinden. Üblicherweise müsste er dies allerdings "reell " nennen, weil kein intentionaler, transzendenter Gegenstand konstituiert wird. Man kann diese Redeweise vielleicht auch so deuten, dass das wich bin bewusst" selbst die Gefühlserfahrung als Realität auftreten lässt (d.h. als Sein, als Wirklichkeit und nicht als blosse Möglichkeit oder Phantasie).

${ }^{50}$ Vgl. für eine genauere Auseinandersetzung mit diesem Thema meinen Aufsatz: Sophie Loidolt, Husserls phänomenologische Kritik der Kantischen Anschauungsformen und die "Widerlegung des Idealismus" - Methodischen Divergenzen, sachliche
} 
heisst "Gegenstand-für-Sein«. Und Bewusstsein hat eben den Vorzug, Gegenstand für sich selbst sein zu können:

... auch es ist undenkbar ohne das wirkliche Subjekt, das Subjekt seiner möglichen Erkenntnis ist. Aber es hat die Auszeichnung, dass es wesensmässig als Ego nur ist, indem es für sich selbst ist, und [...] damit zugleich wirkliches Subjekt der Erkenntnis für sich selbst [ist], also für sich selbst zum Erkenntnisobjekt [wird]. . $^{1}$

Die Einheit des Bewusstseins beruht also trotz der Überlegungen $\mathrm{zu}$ Ich und Nicht-Ich nicht auf irgendeinem "Aussen" (wie es bei Kants empirischem Bewusstsein der Fall ist), wodurch das "Dasein Gegenstände im Raum ausser uns « als bewiesen angesehen wird. ${ }^{52}$ Das Für-sich-sein, das auch ein Nicht-Ich einschliesst, bleibt das entscheidende Merkmal.

Fazit: Die Absolutheit des Bewusstseins beruht auf seiner Gegebenheitsweise, die Sein versichert, nicht auf seiner absoluten Ichheit. In dieser absoluten Gegebenheitsweise kann sehr wohl ein Nicht-Ich notwendig mitgegeben sein. Die These über die Absolutheit des Bewusstseins ist also nicht zu verwechseln mit der Nicht-Absolutheit des Ichs. Dies ermöglicht aber auch gerade Husserls gleichzeitigen Realismus und Idealismus: Eine wahre Welt errichtet sich auf dem Grunde von vorgegebenem Ichfremden. So zeigt sich durch das Medium der reellen Immanenz intentional eine Transzendenz. Doch dieses Ichfremde ist "im Sein", ist "bewusst" dadurch, dass es "für-Sein" ist, auf reeller wie auf intentionaler Ebene (und dies bezeichnet Husserl in beiden Fällen als reelle oder intentionale »Immanenz«).

"Ferner, es gibt im Objektiven eine Stufenfolge, nämlich 1) das Objektive, das als Immanentes schon zum inneren Bewusstsein gehört und das innerlich Ichfremdes bewusst macht: die Hyle und die hyletische Sinnlichkeit, 2) das Objektive, das als Transzendenz Objekt ist der äusseren Sinnlichkeit, von intentionalen Erlebnissen, die selbst im inneren Bewusstsein bewusst sind, aber nur in dieser mittelbaren Weise, und zwar nur durch 'Erscheinungen bewusst sein kann, nämlich als Einheit mannigfaltiger Erscheinungen. Hierbei dienen hyletische Daten als

Konvergenzen, in: Immanuel Kant, Freiheit, Vernunft, Sinnlichkeit - vielstimmiger Widerhall, hg. v.Violetta L. Waibel, Berlin 2022 (im Erscheinen).

${ }^{51}$ Husserl, Einleitung, 277f.

${ }^{52}$ Immanuel Kant, Kritik der reinen Vernunft 1, hg. v. Wilhelm Weischedel, Frankfurt a.M. 1974, 274ff. 


\begin{abstract}
>Abschattungen<, als Träger für `Auffassungen<. Mittels der immanenten ichfremden Objekte als Urobjekte konstituieren sich für das Ich transzendente, als abschattend erscheinende und durch Erscheinungen vermeinte. $\mathrm{Ob}$ es für jedes Ich eine Notwendigkeit ist, dass es transzendente Objekte bewusst hat als seiend oder vermutlich seiend, möglicherweise seiend vermeinte, das muss offen bleiben. $\ll^{53}$
\end{abstract}

Man muss hier natürlich sehr achtgeben, dass der Unterschied zwischen immanent und transzendent nicht verschwindet, auch wenn Husserl von »Urobjekten« spricht. Der Unterschied bleibt aufrecht, dass das Reelle absolut gegeben ist, das intentional Konstituierte, Reale nicht, dass ich mich daher über einen Gegenstand täuschen kann, über ein hyletisches Datum aber nicht, bzw. präziser formuliert: dass ich mich über die Erscheinung eines Dinges täuschen kann, nicht aber über das Erlebnis der Dingerscheinung, das, abstraktiv gesprochen, aus noetischen und hyletischen Komponenten besteht. Wäre dem nicht so, dann wäre die Welt tatsächlich vom "Stoff» des Bewusstseins. Insofern, um zu Jansens $s^{54}$ Ausgangsargument zurückzukommen, beweist ein hyletisch-reelles Nicht-Ich überhaupt keine epistemische oder ontologische Dignität von Realität (genausowenig, wie es die Absolutheit von Bewusstsein abschwächt). Von "Realität" kann ich aufgrund des reellen Nicht-Ichs noch gar nicht sprechen. Nicht nur deshalb, weil sich eine reale Welt aufgrund blosser reeller nichtichlicher Bestände nicht notwendig konstituieren muss (siehe Zitat Husserl oben). Sondern auch, weil dann tatsächlich der Unterschied zwischen Reellem und Realem, Hyletischem und Intentionalem verwischt wird. Das Reelle ist nicht die Welt selbst - das wäre Phänomenalismus. Es bildet sie auch nicht $\mathrm{ab}$ - sonst wären wir in einer Bildertheorie gelandet. Aber das sind wir nach Husserl nicht. Nur intentional sich zeigendes Sein kann »Realität« sein und intentional sich Zeigen heisst sich selbst Zeigen, sich Manifestieren: "Das wahre Sein, und speziell etwa das wahre Sein der Natur, ist nicht ein Zweites neben dem bloss intentionalen Sein. ${ }^{55}$

Vom Vorkommen eines reellen Nicht-Ich auf die "Nicht-Überflüssigkeit» von »Realität« zu schliessen, wie es Jansen ${ }^{56}$ vorsichtig formuliert, was heisst das genau? Es kann doch nicht heissen, dass das reelle Nicht-Ich irgendwie darauf hinweist, dass das intentional sich ausweisende Reale irgendwie das Bewusstsein immer schon

\footnotetext{
${ }^{53}$ Husserl, Zur Phänomenologie der Intersubjektivität (Zweiter Teil), 51.

${ }^{54}$ Jansen, On Transcendental, 29.

${ }^{55}$ Husserl, Einleitung, 276.

${ }^{56}$ Jansen, On Transcendental, 29.
} 
affiziert hat. Denn dann müssen wir es als kausal affiziert denken von etwas, dessen Sein und dessen Affektionsart sich erst als Bewusstseinszusammenhang zeigen, und befinden uns in mindestens so vielen Schwierigkeiten wie Kant bei dem Gedanken einer Affektion durch das Ding an sich (worauf bekanntermassen schon Jacobi hingewiesen hat). Anders formuliert: Aus reell immanentem Nicht-Ich kann man nicht auf eine Affektion von "Aussen« schliessen, weil das transzendental gefasste Bewusstsein kein "Aussen« mehr hat. Und dies hat es deshalb nicht, weil alles, was als "Aussen", als Gegenstand, Sinn macht, sich intentional konstituiert. ${ }^{57}$

\section{Zu Ich und Nicht-Ich als "Gestaltbedingung « des Erscheinens/Seins von Bewusstsein}

Damit komme ich auf den Ausgangspunkt meiner Überlegungen zurück: Transzendentalphilosophie bei Kant beinhaltet, im Gegensatz zu Husserl, Schranken. Durch seine Konzeption der zwei Erkenntnisquellen Anschauung und Begriff (bzw. Sinnlichkeit und Verstand) sowie der notwendigen Verschränkung beider im Begriff der Erfahrung, kann Kant jede Erkenntnis, die bloss auf "leeren Begriffen " beruht, delegitimieren (vor allem dadurch, dass er deren tatsächliche intrinsische Widersprüchlichkeiten aufzeigt). Will man also bei der Erkenntnis bleiben und sich nicht sinnlosen metaphysischen Spekulationen hingeben, dann darf man nicht hinter die Verschränktheit von Anschauung und Begriff zurückgehen. Man erlangt empirische Erkenntnis, wenn man aus empirischen Anschauungen und Begriffen Erfahrungserkenntnis zieht. Man erlangt transzendentale Erkenntnis, wenn man das Zusammenspiel von reinen Anschauungen und Begriffen untersucht. Transzendentalphilosophie soll Ontologie, metaphysica generalis, und metaphysica specialis ersetzen. Eine der Grundüberzeugungen von Kants Transzendentalphilosophie ist, dass man erkenntnismässig nicht über die Verschränkung von Anschauung und Begriff hinauskann (mit dem "Denken" und der praktischen Vernunft allerdings sehr wohl).Vernunft hat sich hier selbst zu kritisieren, sich in ihrem Begehren einzuschränken und die Sinnlosigkeit ihrer theoretischen Wünsche einzusehen.

\footnotetext{
57 Tatsächlich gibt es ein wirkliches "Aussen« des absoluten Bewusstseins, das nicht auf Konstitution angewiesen ist, nämlich die Anderen. Aber selbst diese weisen sich aus und zeigen sich im Ur-Ich. Die absolute Transzendenz zeigt sich also auch in der Immanenz und nirgendwo sonst.
} 
Mir scheint, ein ähnlicher Geist schwebt auch über manchen Auslegungen von Husserls transzendentaler Phänomenologie - bei denen, die zwar einen transzendentalen Idealismus Husserls bejahen, aber mit dem sogenannten "metaphysischen ${ }^{58}$ Idealismus Probleme haben. Und obwohl Husserls Grundkonzeption natürlich eine ganz andere ist, scheint sich eine analoge Idee der transzendentalen Unhintergehbarkeit in der Figur der Verschränktheit von Ich und Nicht-Ich anzubieten. Man könnte dies, angelehnt an MerleauPonty ${ }^{59}$ vielleicht so formulieren: "Es ist sinnlos, über die Gestalthaftigkeit der Gegebenheit von Bewusstsein/Welt, Ich/Nicht-Ich hinauszugehen. Weder kann man den >Hintergrund Bewusstsein verabsolutieren, noch den /Vordergrund Welt (oder umgekehrt). Deshalb ist es auch ein sinnloses Unternehmen, sagen zu wollen, was der ıletzte Seinsgrund ist. Beschäftigen wir uns lieber mit der Analyse der Verflochtenheit von Bewusstsein und Welt bzw. Ich Andere - Welt. Darüber hinaus gibt es nichts Sinnvolles zu sagen.» Man könnte dies vielleicht die transzendental-erkenntnistheoretische Figur der Phänomenologie nennen, im Sinne der Kantianischen transzendentalen Beschränkung metaphysischer Fragen. Damit meine ich einen Ansatz, der mit quasi-Kantischen Schranken operiert und meint, dadurch weitergehende "metaphysische« Überlegungen delegitimieren bzw. für sinnlos erklären zu können.

Erstens glaube ich nicht, dass Husserl trotz aller genetischer Untersuchungen dieser Meinung war. Dies belegt nicht nur das aussagekräftige Zitat aus den Cartesianischen Meditationen in der Einleitung dieses Aufsatzes (man könnte auch noch viele andere anführen). Obwohl sich Husserl in seinen späteren Jahren sicherlich stärker der Verwobenheit von Ich, Anderen und Welt gewidmet hat, hat ihn das m.E. nie dazu bewogen, die "Absolutheitsthese« und die "Abhängigkeitsthese« aufzugeben. Beides schliesst einander nämlich nicht aus.

\footnotetext{
${ }^{58}$ Ich habe diesen Ausdruck bisher nicht verwendet, weil er oft mehr Kontroversen und Abwehrreaktionen hervorruft als produktive Diskussionen. M.E. fällt Husserls "transzendentaler" Idealismus mit seinem "metaphysischen" zusammen, da er von Anfang an beide Elemente aufweist (vgl. Loidolt, Transzendentalphilosophie). Deshalb scheint es mir auch unmöglich, sich entweder für das eine oder das andere zu entscheiden. Ausser natürlich, man versteht als "metaphysisch" nur das, was Husserl ablehnt (siehe Zitat am Anfang, nämlich psychologischen Idealismus und Phänomenalismus) - was ich für einen zu einschränkenden Gebrauch des Begriffs "metaphysisch « halte, den Husserl selbst nicht pflegt. Aber es liegt mir wenig daran, einen Kampf um diese Begriffe und "Schubladen« zu führen. Entscheidend ist nur, wie man die inhaltlichen Thesen versteht.

${ }^{59}$ Merleau-Ponty, Phänomenologie der Wahrnehmung.
} 
Zweitens scheint mir die sachliche Möglichkeit einer Limitierung einfach nicht gegeben. Wie ich versucht habe zu zeigen, ändert das mit dem Ich verflochtene reelle Nicht-Ich überhaupt nichts an der absoluten Gegebenheitsweise von Bewusstsein, auch nicht, wenn sich dadurch sogar in der tiefsten Zeitigung ein ichfremder Kern zeigt. Es ist ein Missverständnis, dass Absolutheit totale Ichheit und noch dazu Ichheit als totale An-Sichheit bedeutet. Das Andere des Ich, das Ichfremde, gehört zum Geschehen von Bewusstsein dazu ${ }^{60}$ (auch in der tiefsten Selbstphänomenalisierung des Flusses). In schlimme Widersprüche verstrickt man sich auf jeden Fall, wenn man dem reellen hyletischen Nicht-Ich eine Affektion durch eine bereits reale Welt hypostasiert.

Drittens: Nur weil Bewusstsein reell als Gestalt von Ich und Nicht-Ich auftaucht, leitet sich daraus nicht eine ebensolche, gleichgeartete gestalthafte Verschränktheit von Bewusstsein und Realität ab. Man kann das eine nicht einfach mit dem anderen gleichsetzen, will man nicht den Unterschied zwischen "reell« und »intentional/ real« über Bord werfen. Reell mag sich eine solche Verschränktheit zeigen und möglicherweise deutet dies auch auf eine Grenze in der Analysefähigkeit hin - das betrifft aber Bewusstsein selbst, in seiner Zeitkonstitution, in seinen reellen Komponenten. Aber intentional bleibt es dabei, dass sich Reales korrelativ zu intersubjektiven, sich ausweisenden Bewussteinsgestaltungen konstituiert und insofern "relativ" auf diese ist. Hier kann ich beim besten Willen keine Schranke sehen, die verbieten würde, auf das absolute Sein des Monadenalls als "tragenden Seinsgrund " zurückzugehen. ${ }^{61}$

\footnotetext{
${ }^{60}$ Ohne hier auf Merleau-Ponty genauer eingehen zu können, scheint dieser mir das Bewusstseinsgeschehen in ein ontologisches Geschehen von Welt zu verlegen. In seiner späten Ontologie spricht er vom "Fleisch der Welt« und von einer »Verschränkung" (Chiasmus) des "Innen" und "Aussen«, von Bewusstsein und Natur, womit er sich der Identitätsphilosophie Schellings annähert (vgl. Maurice Merleau-Ponty, Das Sichtbare und das Unsichtbare, München 1994). In jedem Fall ist diese Lösung, so konsequent sie auch durchdacht sein mag, auch nicht "einfacher" zu verstehen als Husserls transzendentalphänomenologischer Idealismus und wird wohl der naiven, der metaphysischen oder der spekulativen Realistin ebenso viele Probleme bereiten (vgl. Loidolt, Zu den metaphysischen Urtatsachen).

${ }^{61}$ Denn die transzendental beschränkende Behauptung, dass man über das eine nicht sprechen könne, ohne über das andere zu sprechen, trifft nicht ganz zu und ist, wenn, dann keine gleichwertige Reziprozität: Dass man prinzipiell über Realität nicht sprechen kann, ohne über Bewusstsein von Realität (oder Sprache, Begriffe etc.) zu sprechen, scheint für "Korrelationistinnen", Anti-Realistinnen, Transzendentalphilosophinnen aller Art klar zu sein. Dass man aber umgekehrt genauso prinzipiell über Bewusstsein nicht sprechen könne, ohne über Realität zu sprechen, halte ich im phänomenologischen, Husserl'schen Kontext für eine nicht richtige Behauptung, da sich
} 
Viertens erscheint mir eine erkenntnistheoretische Beschränkung ontologischer Fragen, so sinnvoll sie bei Kant ist, im phänomenologischen Kontext zu kurz zu greifen (ausser freilich, man versteht Husserl so, dass er nur Aussagen über möglichen Sinn, aber nie über Sein und Wirklichkeit macht, was mir verfehlt erscheint). Phänomenologie ist nicht nur Erkenntnistheorie, die keine ontologischen Aussagen macht.Vielmehr verknüpfen sich in der Phänomenologie transzendentale Aussagen über Erkenntnisbedingungen mit Aussagen über Seinsbedingungen. Wenn Kant von Anschauung und Begriff, Rezeptivität und Spontaneität spricht, dann handelt es sich um zwei Erkenntnisquellen, hinter deren Verschränkung man nicht zurückkann. Mir scheint es schwierig, dies auf die Korrelation Bewusstsein - Welt zu übertragen. Warum? Wenn man tatsächlich behaupten will, dass sich der Gegenstand selbst manifestiert und nicht nur seine Erscheinung; wenn man sich weiterhin der These verpflichtet sieht, dass intersubjektiv ausgewiesenes, sich bewährendes Erscheinen nichts anderes ist als reales Sein, hinter dem sich kein An-Sich mehr zurückzieht - dann kann man auch die Korrelation Bewusstsein - Welt nicht bloss erkenntnistheoretisch lesen.Vielleicht aber doch die reelle Gestalt Ich/Nicht-Ich? Vielleicht ja. Aber dies ist keineswegs mit dem Verhältnis Bewusstsein - Welt kurzzuschliessen, wie ich oben argumentiert habe.

Doch eventuell liegen die Dinge ja noch etwas anders. Vieles an Julia Jansens Position erinnert an Robert Sokolowskis `Klassiker The Formation of Husserl's Concept of Constitution (1964), in dem dieser zu der Formulierung gelangt, dass Bewusstsein zwar eine notwendige, aber keine hinreichende Bedingung für Sinn, Sein und Realität sei. ${ }^{62}$ Und bei Sokolowski klingt dies eher ontologisch als transzendentalerkenntnistheoretisch: »[P]henomenology studies subjectivity as the condition of possibility for the emergence of sense and reality, not as the adequate cause and sufficient reason for what is constituted. $\aleph^{63}$ Dies ist natürlich absolut richtig, insofern Sokolowski betont, dass Husserl der Rolle der Faktizität Raum gebe. Aber Husserl hat auch niemals behauptet, dass sich alles aus dem transzendentalen Ego ableiten liesse. Es lässt sich sogar noch viel weniger daraus ableiten als bei Kant, wo in der transzendentalen Subjektivität der kategoriale

trotz aller Anerkennung des Urfaktums von Weltgegebenheit die Denkmöglichkeit ergibt, dass Weltkonstitution zusammenbricht. Das ist und bleibt kein Widersinn, so wie es umgekehrt sehr wohl einen Widersinn ergibt, Welt jenseits von Gegebenheit zu denken.

${ }^{62}$ Robert Sokolowski, The Formation of Husserl's Concept of Constitution, Dordrecht 1970, 197.

${ }^{63}$ Ebd., $192 \mathrm{f}$. 
Apparat quasi fix vorliegt, während er bei Husserl aus der vorprädikativen Gegebenheit durch prädikative, kategoriale Akte gewonnen wird. Alles Empirische und Eidetische ist vielmehr Gegebenheit, Vorgegebenheit, Affektion, an der sich das Ego betätigt. Dass das Ego also der Emanations- und Entstehungsgrund allen Seins im Sinne Gottes wäre ${ }^{64}$ ist eine Befürchtung, die nie auf Husserls Idealismus zugetroffen hat. Dennoch ist Bewusstsein der "Seinsgrund " dessen, was ihm passiv vorgegeben ist. Diesbezüglich scheinen mir Husserls Aussagen bezüglich "hinreichend « und "notwendig» recht eindeutig:

"Ist in seiner Weise der Bewusstseinsfluss, so ist alles, was sonst ist und irgend sein kann. Es bedarf keines weiteren. Und ist irgendetwas, was wir sseiend aber nicht `Bewusstsein nennen, so ist Bewustsein, und eben solches Bewusstsein, das keines weiteren bedarf, um zu sagen 'Es ist das Seiender. Und dieses ।Es ist sagt: Es ist im Bewusstseinsfluss, aus ihm herauszunehmen als darin geborgene, darin wurzelnde Einheit. ${ }^{65}$

Wenn man also die "Abhängigkeitsthese" (dependency-thesis) im kreationistischen Sinn liest, dass Bewusstsein Welt hervorbringt, dann lässt sich Sokolowskis Formulierung von der notwendigen aber nicht hinreichenden Bedingung gut verstehen. Aber damit haben wir ja nur eine absurde Deutung von Husserls Idealismus abgewehrt, die er selbst nie vertreten hat. Was seine positive Bedeutung ist, verstehen wir damit noch keineswegs. Und diese beinhaltet zunächst einmal, dass bezüglich eines Seinsgrundes, der kein Hervorbringungsgrund ist, Husserl ganz deutlich auf der hinreichenden Bedingung von Bewusstsein besteht (auf keinen Fall kann man sagen, Bewusstsein bedinge nur dem Erscheinen aber nicht dem Sein nach). Genügt die erkenntnistheoretische Berufung auf die reelle Bewusstseinsgestalt von Ich und Nicht-Ich, um diese These als »sinnlos" abzuwehren?

Wenn nicht, haben es diejenigen, die sich noch immer auf die Figur des "notwendig aber nicht hinreichend « berufen wollen, dann statt mit zwei "Erkenntnisquellen« mit zwei »Seinsquellen ${ }^{66}{ }^{6} \mathrm{zu}$ tun? Und was soll das heissen? Ich muss diese Fragen hier unbeantwortet

\footnotetext{
${ }^{64}$ Husserl sagt in den Ideen I deutlich, dass das Absolute Gottes »ein >Absolutes in einem total anderen Sinne als das Absolute des Bewusstseins" wäre (Husserl, Ideen I, 125).

${ }^{65}$ Husserl, Transzendentaler Idealismus, 70.

${ }^{66}$ Dagegen, dass dies nicht auch Sein von der Art des Bewusstseins wäre, spricht im Grunde schon dies: "Es ist auch klar, dass es hinter dem absoluten Bewusstsein kein neues Sein geben (kann) und dass alles, von dem wir mit Sinn und Recht sollen sagen können: `Es ist‘, im Bewusstsein (sich) Konstituierendes oder in Beziehung auf Konstituiertes durch Abstraktion Gewonnenes sein muss. (Denn allgemeine Gegenstände und Ideen, wenn auch nicht auf wirkliches Bewusstsein zurückweisend, weisen of-
} 
stehen lassen. Doch immerhin eines muss klar sein: "Woher« auch immer das Nicht-Ich »kommt", es kann nicht durch Affektion aus einer Natur kommen, die so ist wie konstituierte Natur, aber ihr der Sache nach vorangeht. Und: Es kann nicht aus einer Entität herstammen, die prinzipiell unerkannt bleibt (wie das Ding an sich). Dies schränkt m.E. den Antworthorizont zumindest schon einigermassen ein.

\section{Urfaktizität, Realismus und Idealismus: ein Kippbild?}

Husserls Antwortversuche bezüglich dieser "letzten Fragen" sind bekannt. Es gibt Spekulationen über die Teleologie des Monadenalls, über Gott (was meist mit dieser Teleologie zusammenfällt), sogar über panpsychistische Varianten (dies eher selten). ${ }^{67}$ Keine dieser phänomenologischen Spekulationen darf als besonders überzeugend gelten und Husserl ist sich dessen selbst bewusst. Am ehesten ist seine Phänomenologie der Urtatsachen, die László Tengelyi ${ }^{68}$ zu einer "Metaphysik der zufälligen Faktizität" ausgebaut hat, ein phänomenologisch tragfähiger Anhaltspunkt. "Zufällig", um keine Missverständnisse zu erzeugen, ist dabei aber nur die Urtatsache der Welthabe selbst - die weiter auf Intersubjektivität, d.h. auf ein intentionales Ineinander pluraler Monaden als »die smetaphysischer Urtatsache ${ }^{69}$ verweist. Husserls phänomenologische Metaphysik der Urfaktizität besagt, dass es das Urfaktum der Welthabe, nämlich einer realen, raum-zeitlich konstituierten Welt, braucht, damit es überhaupt jemals zu irgendwelchen Wesenseinsichten kommen kann. Damit ruht letztlich alle Eidetik auf diesem kontingenten Urfaktum, das Husserl (und Tengelyi) als faktische Notwendigkeit verstehen. Andererseits impliziert gleichzeitig das Urfaktum dieser meiner Welthabe, dass diese und jene Entwicklungen teleologisch und wesensmässig notwendig waren, sodass eben genau diese Welt ist. Husserl geht hier sehr weit damit, Entwicklungsnotwendigkeiten $\mathrm{zu}$ behaupten. Eine der zentralen, weiter gefassten Einsichten ist auf jeden Fall, dass sich eine objektive Welt nur durch verleiblichte

fenbar auf mögliches hin, und die Möglichkeit ist schliesslich selbst nur denkbar in Beziehung auf aktuelles Bewusstsein.)《 (Husserl, Transzendentaler Idealismus, 72.)

${ }^{67}$ Vgl. Edmund Husserl, Grenzprobleme der Phänomenologie. Texte aus dem Nachlass (1908-1921), Dordrecht 2014.

${ }^{68}$ Tengelyi, Welt und Unendlichkeit.

${ }^{69}$ Husserl, Zur Phänomenologie der Intersubjektivität (Dritter Teil), 366; vgl. Tengelyi, Welt und Unendlichkeit, 185. 
Monaden konstituieren kann. Wie bereits erwähnt, führt die Analyse der Urfaktizität Husserl problemlos auf eine letzte metaphysische Urtatsache, deren Absolutheit er keineswegs ausweicht: das "Ineinander des Absoluten". Ein diesbezügliches Zitat bringt uns auch noch einmal auf die Problematik des Nicht-Ich:

"Aber wenn so alles in mir intentional beschlossen ist, was überhaupt denkbar ist und möglicherweise und wirklicherweise ist, so sagt das nicht, dass alles, was ist, mein transzendentales Ich allein ist. Alles Nicht-Ich lliegt selbst im Ich, aber als intentionale Einheit der Geltung, obschon als `Transzendenz nicht Ich. So liegt auch das andere transzendentale Ich in mir, es liegt in mir so als Geltungseinheit, dass es antizipierte und bewährte Seinsgewissheit ist, und zwar als das Nicht-Ich, das selbst Ich ist und das als anderes Ich mich selbst wiederum in sich trägt. Diese Innerlichkeit des Füreinanderseins als eines intentionalen Ineinanderseins ist die rmetaphysische Urtatsache, es ist ein Ineinander des Absoluten. ॥ $^{70}$

Wenn es also ein Nicht-Ich gibt, das sich als radikale Transzendenz und als absolutes Für-Sich manifestiert, dann sind es die Anderen. Hingegen: "Die Transzendenz eines Naturobjektes ist eine grundwesentlich andere als die Transzendenz eines fremden Subjekts, einer fremden monadischen Subjektivität. « ${ }^{71}$ Husserl spricht sogar von einem "abgrundtiefen Unterschied «. ${ }^{72}$ Dies deshalb, weil Natur sich als Transzendenz eines seinsmässig Relativen bekundet (und das bereits in einer Monade). Nur »intersubjektiv-transzendentale Sozialität $\aleph^{73}$ ist absolut.

Bleiben wir also bei der Faktizität des Affektionsgrundes einfach stehen - was doch immerhin einige weitgehende Einsichten erlaubt? Ich betone es ein letztes Mal: Dies kann man machen, solange man nicht in eine Kantische Ausdeutung dieses »unbekannten Affektionsgrundes" verfallt. Und dies behaupte ich nicht deshalb, weil ich eine Kantische Position für falsch halte, sondern nur, weil ich sie für nicht vereinbar mit gewissen zentralen Husserl'schen Einsichten halte. Es wird sich also alles darum drehen, Husserls Faktizität von der Kants zu unterscheiden. Husserl ist auch nicht Fichte, das Ich setzt sich nicht sein Nicht-Ich. Und transzendentales Bewusstsein ist, wie nun noch einmal klar wurde, nicht "allein", "solipsistisch", sondern

${ }^{70}$ Husserl, Zur Phänomenologie der Intersubjektivität (Dritter Teil), 366.

${ }^{71}$ Husserl, Zur Phänomenologie der Intersubjektivität (Zweiter Teil), 244.

${ }^{72}$ Husserl, Cartesianische Meditationen, 35.

${ }^{73}$ Ebd. 
im Gegenteil, als Absolutes transzendental vergemeinschaftet. Natürlich drängt sich uns die Frage auf, "woher« denn die Affektion, das Ichfremde in der Grundgegebenheit von Ich/Nicht-Ich "kommt«. Doch "woher" kommt das Ich, das es ja "allein" auch nicht gibt? Eine phänomenologische Metaphysik der Urtatsachen lässt uns zumindest diese philosophisch ausloten, ohne jedoch auf das "Woher» der Faktizität eine Antwort zu haben.

Man kann schliesslich auch noch eine ganz andere Perspektive auf die Sache der Urfaktizität haben: nämlich die empirische, dass die Welt einfach "ist" und sich uns zeigt und wir versuchen, sie ausgehend von der Lebenswelt bis in ihre kleinsten physikalischen Bestandteile mit intentional motivierten Konstruktionen wissenschaftlich zu erfassen. Was will man mehr von einem Realismus? Was sich uns zeigt, können wir nicht bestimmen, uns nicht aussuchen. Wir bringen es nicht hervor. Vorgegebenheit und Idealismus machen einen empirischen Realismus möglich. In der Metaphysik der Urtatsachen hat sogar die Apodiktizität des cogito nur Sinn auf dem Boden der Weltgeltung ${ }^{74}$ - was bedeutet, dass urfaktisch eben eine Welt existiert und ich deshalb mich urfaktisch als ego cogito erfahre.

"So enthüllt sich der phänomenologische Idealismus als eine transzendental-phänomenologische Monadologie, die nur keine metaphysische Konstruktion ist, sondern eine systematische Auslegung des Sinnes, den für uns alle vor allem Philosophieren die Welt hat, ein Sinn, der nur philosophisch entstellt, aber nicht geändert werden kann. $\|^{75}$

Husserls transzendental-metaphysischer Idealismus heisst nicht, dass da nichts ist ausser Geist. Er behauptet vielmehr, dass sich nichts zeigen und nichts sein kann ohne Bewusstsein. Und weiter: Dass Sein nicht denkbar ist ohne prinzipielle, motivierte, reale Möglichkeit, sich einem Bewusstsein zu zeigen. Den Seinsbegriff davon zu trennen ist: Widersinn. ${ }^{76}$ Bei allen Schwierigkeiten: Mir ist keine

\footnotetext{
${ }^{74}$ Edmund Husserl, Zur phänomenologischen Reduktion. Texte aus dem Nachlass (1926-1935), Dordrecht 2002, 469.

${ }^{75}$ Husserl, Cartesianische Meditationen, 36.

${ }^{76}$ Im Grunde scheint mir der Konflikt zwischen Realismus und Idealismus ein Konflikt von Intuitionen zu sein, die sich beide aus dem Sinn von "Objektivität» bzw. "Realität " ergeben. Die Idealistin beruft sich auf das Sinnargument (was meinen wir eigentlich mit "sein", "existieren", "Objektivität" etc.?), d.h. auf Einsichten, die uns zeigen, was denkbar ist und was auf Widersinn führt, was letztlich auf die Inanspruchnahme der Identität von Sein und Denken hinausläuft. Im Sinn von Objektivität und Realität steckt nun aber das Paradox, dass objektives Sein einerseits nur als Gegebensein denkbar ist (bzw. Wahrheit immer nur "für uns" ist und "Sinn macht»), während es
} 
philosophische Position bekannt, die keine Schwierigkeiten verursacht, aber wenige, die so klar durchdacht und argumentiert sind. Anstatt also zu sagen: Bewusstsein ist aufgrund der Ich/Nicht-IchStruktur vielleicht doch nicht so "absolut", und vielleicht lässt sich dadurch den allzu idealistischen Tendenzen entkommen, würde ich eher dafür plädieren, weiterhin plausibel zu machen, warum sich Realismus und Idealismus bei Husserl wie bei einem Kippbild ${ }^{77}$ miteinander vereinbaren lassen. Vielleicht gehören Realismus und Idealismus im Husserl'schen Sinn in ihrer Urfaktizität sogar gestalthaft zusammen. Deshalb handelt es sich ja, wie er betont, auch nicht um einen Siegespreis, den man aus dialektischen Spielen gewinnen könnte.

- Prof. Dr. Sophie Loidolt ist Lehrstuhlinhaberin für "Praktische Philosophie» am Institut für Philosophie der TU Darmstadt und Mitglied der "Jungen Akademie« der Österreichischen Akademie der Wissenschaften. Ihre Forschungsschwerpunkte liegen in der Phänomenologie, der politischen Philosophie, der Rechtsphilosophie und Ethik sowie in der Transzendentalphilosophie und der Philosophie des Geistes.

andererseits bedeutet, unabhängig von Gegebensein und Sinnerfassung zu sein. Dieses Grundparadox lässt sich nur weiter explizieren, aber nicht auflösen. Die Realistin macht die Teilthese der radikalen Unabhängigkeit zu ihrer Grundintuition (und verstrickt sich dadurch in die Widersprüche des metaphysischen Realismus). Während die Idealistin das rationale Argument eher auf ihrer Seite hat (durch die Intuition einer Konvergenz von Sein und Denken), hat die Intuition der Realistin eine Art "naturwüchsiger Stärke«. Ich denke, dass Husserl versucht, beiden Intuitionen gerecht zu werden und sie zu versöhnen. Sie bloss gegeneinander auszuspielen hiesse auf jeden Fall, der Spannung im Sinn von Realität und objektivem Sein selbst nicht zu entsprechen.

${ }^{77}$ Bei Kant hingegen würde die Metapher des "Kippbildes« nicht funktionieren, obwohl er durchaus betont, dass wir "Bürger zweier Welten" sind - was sich auf die Husserl'sche "mundane " und "transzendentale« Subjektivität eventuell übertragen liesse. Während bei Husserl aber durch "Blickwendung" oder "Einstellungswechsel" das transzendentale, absolute Bewusstsein jederzeit in den theoretischen Blick kommen kann, bleibt für Kant das Noumenale unergründlich und ist nur durch die praktische Vernunft in uns als Aufruf zum vernünftigen/moralischen Handeln installiert. 